\title{
CORRIGENDUM
}

\section{Widespread distribution of proteorhodopsins in freshwater and brackish ecosystems}

Nof Atamna-Ismaeel, Gazalah Sabehi, Itai Sharon, Karl-Paul Witzel, Matthias Labrenz, Klaus Jürgens, Tamar Barkay, Maayke Stomp, Jef Huisman and Oded Beja

The ISME Journal (2010) 4, 462; doi:10.1038/ismej.2009.130

Correction to: The ISME Journal (2008) 2, 656-662; doi:10.1038/ismej.2008.27

Since the publication of this paper, the authors have noticed an error in Table 2. The correct table is shown below.

Table 2 The proteorhodopsin forward and reverse primers used in this study, designed based on known PR sequences found in the NCBI and Global Ocean Sampling datasets

\begin{tabular}{ll}
\hline Forward & Reverse \\
\hline RYIDW (5'-MGNTAYATHGA & GWAIYP (5'-GGRTADATNGCCC \\
YTGG-3') & ANCC-3') \\
& GWLIYP (5'-GGRTADATNARCC \\
& ANCC-3') \\
& GWVIYP (5'-GGRTADATNACCC \\
& ANCC-3') \\
& GWIIYP (5'-GGRTADATDATCC \\
RYADW (5'-MGNTAYGCNGA & ANCC-3') \\
YTGG-3') & GWSIYP (5'-GGRTADATNSWC \\
& CANCC-3') \\
& GWGTYP (5'-GGRTANGTNCCC \\
& CANCC-3') \\
& GWAVYP (5'-GGRTANACNGCC \\
RYFDW (5'-MGNTAYTTYGA & CANCC-3') \\
YTGG-3') & GWLVYP (5'-GGRTANACNARC \\
& CANCC-3') \\
& GWVVYP (5'-GGRTANACNACC \\
& CANCC-3') \\
& GWGVYP (5'-GGRTANACNCCC \\
RYVDW (5'-MGNTAYGTNGA & CANCC-3') \\
YTGG-3') & GWSVYP (5'-GGRTANACNSWC \\
& CANCC-3') \\
& GWIVYP (5'-GGRTANACDATCC \\
& ANCC-3') \\
&
\end{tabular}

Variable amino acids in the translated primer regions are marked in bold letters.
The authors would like to apologize for any inconvenience this may have caused. 